\title{
Desarrollo de la prensa en Ecuador. De la prensa ideológica a la empresa periodística
}

\section{Development of the press in Ecuador. From the ideological press to the journalistic enterprise}

Isidro Marín Gutiérrez, Universidad de Huelva - isidro.marin@dstso.uhu.es

Diana Rivera Rogel, Universidad Técnica Particular de Loja - derivera@utpl.edu.ec

Patricio Barrazueta Molina, Universidad Técnica Particular de Loja - pbarrazueta@utpl.edu.ec

\section{Abstract}

Since the begining like an independent republic in 1830 the relations between diferent goverments and the mass media, specially print, has been conflicting. The papers took a political and no informative actitude. The history have registred several attacks against the liberty and safety of the journalist and media companies. This article summarizes the history of the ecutorian newspapers form it's born like a republic untill media companies are consolidated as stable and permanent industries.

Keywords

Mass media, press, newspaper, laissez-faire, Ecuador.

Resumen

Desde el origen del país como república independiente en 1830 las relaciones entre los gobiernos y los medios de comunicación, especialmente los impresos, ha sido conflictiva. Los periódicos asumieron una actitud política más que informativa. En la historia de Ecuador se han resgistrado varios atentados contra la libertad e integridad de los periodistas así como de las empresas de comunicación. Este artículo resume la trayectoria de los periódicos ecuatorianos desde sus inicios hasta el momento presente en donde las empresas periodísticas están consolidadas como medios de comunicación estables y permanentes.

Palabras clave

Medios de comunicación, imprenta, periódicos, liberalismo, Ecuador.

Sumario

1. Introducción. 2. Diario El Tiempo. 3. Diario La Patria. 4. El periódico vespertino La Ley y El Grito del Pueblo. 5. Los periódicos satíricos de El Buscapié a Fray Gerundio. 6. La consolidación del periodismo de empresa. De El Comercio a El Universo. 7. La huelga general de 1922. 8. El diario El Día y el desarrollo de la prensa regional como El Mercurio de Cuenca. 9. A modo de conclusión. 10. Bibliografía. 


\section{Introducción}

El desarrollo de la imprenta ha sido un símbolo de la modernidad. Se estableció de forma temprana en América y ayudó a la difusión de los libros en las colonias. La primera imprenta llegó a Quito en 1754 y en menos de 40 años se creó la primera prensa. El periódico Primicias de la Cultura de Quito, que apareció el 5 de enero de 1792 (Torre Revello, 1991: 185). Es cronológicamente la tercera cabecera más antigua de América del Sur, precedida de La Gaceta de Lima, en 1743, y El Papel Periódico de Bogotá, en 1791 (Maeder, 2001: 19).

Eugenio Espejo fue uno de los próceres de la independencia quiteña. Él fue uno de los propagadores de las ideas progresistas. Fue el fundador del primer periódico quiteño Primicias de la Cultura de Quito en el que se exponían los ideales libertarios (Punín \& Rivera, 2014:117). El historiador José Torre asegura que Primicias de la Cultura de Quito "desde su iniciación, este periódico fue combatido con encono por émulos y enemigos personales de Espejo, dolidos [por] sus antiguas sátiras e ironías" (Torre Revello, 1991: 186). Los registros históricos constatan que se publicaron siete números de circulación consecutiva. El el último número de este periódico podemos leer a Francisco Eugenio de Santa Cruz y Espejo, precursor de la independencia de Ecuador: "ya somos consocios, ya somos quiteños (quiere decir, no españoles), entramos ya en la Escuela de la Concordia, de nosotros renace la Patria, nosotros somos los árbitros de su felicidad" (De Velasco, 1981, IX). Estaba claro que para las autoridades españolas había llegado la hora de encarcelar a Eugenio Espejo, censurar este periódico y cualquier periódico para no tener problemas. Pero la Indepedencia de España no significó libertad de prensa. En 1825 el periódico llamado El Espectador Quiteño fue asaltado por el general Juan José Flores, intendente general del departamento de Quito (entre 1823-1830) quien rompió la imprenta y arrojó los tipos a la calle (Rolando, 1947: 139).

En Ecuador, desde su nacimiento como República, en 1830, la pugna ideológico-política de movimientos proselitistas que ansiaban la toma del poder, permitió que a nivel de un periodismo altamente concebido se defendieran intereses por un lado y rechazos por otro, con la finalidad de hacer resistencia a los movimientos que estaban empotrados en el poder político-administrativo. Durante el periodo 1830-1845 gobernaron el país Juan José Flores, Vicente Rocafuerte y el triunvirato conformado por José Joaquín de Olmedo, Vicente Ramón Roca y Diego Noboa. Históricamente esta fase política de inicios de la república, no se creó nada nuevo en el ámbito del periodismo, es heredera de la labor que hiciera Eugenio Espejo, desde la segunda mitad del siglo XVIII (Nuñez Endara, 2001).

En 1843 Juan José Flores Aramburu, primer presidente de Ecuador, dictó la "Carta de Esclavitud", que entre otras cosas restringía la libertad de prensa y pretendía eternizarse en el poder. Esta carta provocó revueltas y manifestaciones populares en todo el país. Con el estado ecuatoriano aún en cimientos y con problemas financieros a causa de la guerra de independencia con España, cada gobernante de turno permitió e incluso impulsó la fundación de periódicos que difundían no solo sus obras, sino y sobre todo su doctrina (Montalvo, 1990, 29).

Desde 1833 hasta 1838 se publicó en Guayaquil El Ecuatoriano, que difundió los programas políticos y de desarrollo del presidente Juan José Flores, en contra de Vicente Rocafuerte, pero una vez que éste último ascendió al poder, el periódico cambió de orientación ideológico-política. No ha sido el único caso de un periódico que ha cambiado su línea editorial, acondicionada y sumisa a las propuestas de los gobiernos (Rolando, 1934).

Constituida la República de Ecuador en nación libre e independiente, en mayo de 1830, se publicó en Guayaquil El Colombiano del Guayas (Rolando, 1947). En el mismo año se publicó La Gaceta de Quito, Ocios, Memorias de un Patriota, El Amigo de los Pueblos, El Hombre Libre, La Gaceta del Gobierno del Estado Ecuatoriano, El 9 de Octubre, El Quiteño Libre (cuyo fundador, Francisco Hall, resultó el primer mártir del periodismo ecuatoriano a pesar de ser británico). En 1831 comenzó a publicarse El Patriota. Para 1832 se publicaron los periódicos El Amigo del orden, El Centinela, El Ecuatoriano del Guayas y Las Armas de la Razón, este último se editó en Guayaquil. En 1833 se publicaron El Trece de Febrero y el Investigador en Cuenca (Ayala Mora, 2008, 64). De todos estos periódicos, así como de sus fundadores y periodistas existen escasas huellas en la historia, ya que nacieron con fines partidistas y en una época de limitadas facilidades técnicas que les permitieran consolidarse como medios de comunicación.

Adicionalmente se publicaron en Quito El Monitor Quiteño, Las Facultades Extraordinarias y El Doce de Octubre en 1833. También en la ciudad de Quito salieron a la luz El Argos, Estrella de Lumbisi, El Chihuahua, El Triunfo de la Libertad, El Imbabureño (en Ibarra) y El Patriota (en Cuenca) en 1834. La Voz del Ecuador (Quito), El Convencional del Ecuador (en Tungurahua), y El Semanario Eclesiástico (en Cuenca) comenzaron a publicarse en 1835. El Ariete se publicó por primera vez en 1838. La Verdad Desnuda, El Imparcial (Quito), El Chanduy, El Patriota del Azuay (Cuenca), El Sufragante (Quito), La Balanza, La Opinión, El Ultramontano y El Argos, comenzaron todos ellos en 1839. También se publicaron en Quito en 1839 El Observador con Anteojos, El Amigo de la Sociedad, El Aspirante de la llustración y El Poder de los Principios; y en Guayaquil ese año comenzó El Popular (Rolando, 1947). También se fundaron La Gaceta del Gobierno Provisorio del Ecuador, El Registro Auténtico Nacional de la República del Ecuador, el Registro Municipal y La Opinión en 1839.

Otros periódicos que fueron fundados en la misma época fueron El Meteoro Eleccionario (Quito) en 1840. El Correo Semanal, La Razón (Cuenca), El Faro (Quito) y El Popular comezaron en1841. El Registro de las Actas de la Convención (Quito) sacó su primer número en 1843. La Concordia (Quito), El Seminario Mercantil, La Luz (Cuenca), y El Spleen comenzaron a imprimirse en 1844. El Censor, El Veintiuno de junio, El Patriota de Quito, El Norte del Republicano, El Ecuatoriano, El Seis de Marzo, el Nacional, El Atalaya, Las Armas de la Razón, El Trece de Febrero y Las Facultades Extraordinarias, todos ellos de Quito, comenzaron en 1845 (De Janon Alcivar, 1948).

Uno de los personajes más destacados en el periodismo ecuatoriano fue Gabriel García Moreno quien editó El Zurriago y fundó El Vengador, el primero para combatir al presidente Vicente Ramón Roca Rodríguez, y el segundo contra el presidente Juan José Flores Aramburu (Rolando, 1947). También fundó La Nación para atacar al presidente José María Urbina y Viteri y La Unión Nacional, de tendencia conservadora, en cuyas columnas promocionaba su quehacer político (Ponce, 1990).

Bajo las dos presidencias de Gabriel García Moreno existieron en Ecuador 21 imprentas de las cuales seis estaban en la provincia del Azuay. De todas ellas sólo salían ocho periódicos, debido a la centralización del poder del presidente que gobernaba amparado en la "Carta Negra" (la Constitución de 1869) (Vaca Del Pozo, 1941). Posteriormente gobernaron Ecuador Antonio Borrero Cortázar (presidente 
constitucional entre 1875 y 1876), quien gobernó el país como presidente constitucional y posteriormente como dictador entre 1876 y 1883. Este periodo se caracterizó por el final de la dictadura de Gabriel García Moreno. Políticamente el país se debatía no sólo ante luchas sociales sino también armadas. Es así que en 1876 comenzó un descontento general con la instauración de una sublevación militar dirigida por Ignacio de Veintemilla (Reyes, 1970).

Con el desarrollo del telégrafo, inaugurado en 1888, con 33 oficinas y 1.600 kilómetros de líneas, y el desarrollo del ferrocarril se dio un impulso a las comunicaciones y, entre ellos al periódico. A finales del siglo XIX la prensa ecuatoriana contaba ya con 90 periódicos principalmente diarios, de los cuales 40 se encontraban en la ciudad de Guayaquil (Benítez, 2000). La toma de poder por parte del Partido Liberal, liderado por el caudillo Eloy Alfaro, da un giro de 360 grados en el aspecto social y de las libertades en Ecuador. Las luchas de Eloy Alfaro a través de guerras civiles, enfrentamientos y pugnas de todo calibre, le permitieron organizar una nueva política, una nueva república que incidió mucho en la vida del pueblo ecuatoriano. Después de su asesinato (1911), los partidos conservadores del país creyeron que con su asesinato terminaba la influencia del liberalismo. Pero no ocurrió así, porque los planes y propuestas de la nueva política perduran hasta hoy, sin que gobiernos subsiguientes hayan podido cambiar toda la estructura ideológica que nació en la revolución liberal de 1895 (Iglesias Mata, 1995).

Los periódicos de la época, creados a partir de la visión política de sus fundadores, siguieron los pasos de los gobiernos, poniendo especial énfasis en las actividades de los gobiernos liberales como el de Eloy Alfaro. La prensa tenía una presencia muy importante en casi todo el territorio nacional, y se hizo eco del proceso denominado "Venta de la Bandera". Este escándalo que se suscitó en 1894 se dio cuando China y Japón se encontraban en guerra por el control de Corea, y los estados americanos se declararon neutrales. Aun así Chile consiguió la autorización del gobierno ecuatoriano para que un buque de guerra, cedido por el gobierno chileno a Japón atravesara el Océano Pacífico con la bandera ecuatoriana, esta noticia tuvo como consecuencias la indignación del pueblo ecuatoriano y la dimisión del primer mandatario, la salida del país de Jose María Plácido Caamaño a quien le atribuían la responsabilidad de este hecho. Estos incidentes crearon un ambiente propicio para que un levantamiento liberal en Guayaquil proclamara la jefatura suprema de Eloy Alfaro. El periodista guayaquileño Juan Murillo Miró, exiliado en Chile, consiguió la información y la envió a los medios periodísticos nacionales (Reyes Cedeño, 2000).

El triunfo del liberalismo en el poder en 1895 fue apoyado por la actitud de varios periódicos que mantuvieron constantes luchas contra la reacción conservadora como el periódico El Constitucional, organizado por José Peralta; El Tiempo de Luciano Coral, o El Telégrafo. Todos ellos apoyaban las tesis liberales. Hay que tomar en cuenta que en esta época los periódicos eran más bien órganos de difusión de ideologías y no de noticias. Los periodistas eran escritores, políticos e inclusive los mismos propietarios de los medios apoyando 0 criticando a los gobiernos de turno. Incluso en los propios medios se hacía partícipe la tendencia ideológica a la que pertenecían inscribiendo en las portadas "diario radical" o "diario liberal" (Rivera Rogel, 2012).

Entre 1895 y 1912, durante el liberalismo radical, nacieron 237 periódicos en Guayaquil. La mayoría de corte liberal, muchos de ellos circularon por pocas semanas, ya que su nacimiento tenía que ver más con fines propagandísticos (Gómez Iturralde, 1998). Pese a que Eloy Alfaro promovía la libertad de prensa en Ecuador, hubo una respuesta bastante violenta por parte del gobierno en diarios como El Telégrafo (1884), El Ecuatoriano o La Nación (1900). Todos estos periódicos estaban asentados en Guayaquil. La censura de estos medios se dio después de que hicieran pública su oposición al gobierno alfarista. Uno de los casos más seguidos por la prensa fue el "contrato Harman" (Uggen, 2004). Los medios realizaron una fuerte campaña para impedir que la prórroga que pedía la empresa fuese concedida.

Como consecuencia se devaluaron los bonos (aproximadamente 17 millones y medio de dólares) con los que el gobierno de Ecuador pagaba a la Compañía de Ferrocarriles, lo que deterioró su situación. El gobierno negoció un préstamo externo con la French Finance Corporation, luego de esta negociación más del setenta por ciento de los valores pasaron a Harman quien, pese a sus movimientos inusuales, gozaba de la completa confianza de Eloy Alfaro. Además, como la compañía carecía de solvencia económica, le entregaron en calidad de préstamos fondos destinados al servicio de los bonos de la deuda externa (Corral Burbabo de Lara, 2006). Durante la primera presidencia de Eloy Alfaro los medios de comunicación fueron perseguidos y amonestados, en especial, después del triunfo de la revolución liberal, para consolidar el poder sobre el grupo conservador que era muy numeroso y estaba asentado especialmente en la sierra ecuatoraina. El gobierno alfarista tuvo acciones poco ortodoxas contra los periódicos. El gobierno de Eloy Alfaro fue un gobierno fuerte, con frecuentes inclinaciones hacia la tiranía. Para llegar al establecimiento del liberalismo, tuvo que suprimir muchas libertades esenciales. Se encarceló, se desterró y se clausuraron periódicos (Carrión, 2008). Uno de los hechos que fue recogido por la prensa de la época fue la ejecución del periodista cuencano Víctor León Vivar y Correa, por parte del coronel Manuel Antonio Franco, lugartentiente de Eloy Alfaro. En 1895 escribía en el periódico La Ley donde se expresaba duramente contra el gobierno liberal de Eloy Alfaro. El presidente al tener conocimiento de los escritos ordenó la prisión de sus redactores. La Ley se publicaba en la imprenta de la iglesia con el visto bueno del arzobispo. El 25 de septiembre de 1895 una turba afín al gobierno asaltó la imprenta destruyéndola. Víctor León Vivar en junio de 1896 se alistó en las partidas guerrilleras en contra del gobierno. Para el 5 de agosto fue prendido y ejecutado. Vivar fue uno de los periodistas que más enfrentó las políticas alfaristas y su sistema de gobierno (Pérez Pimentel, 2005).

\section{Diario El Tiempo}

En 1899 apareció en Guayaquil diario El Tiempo, fundado por don José de Lapierre y más tarde editado bajo la dirección de Luciano Coral; un firme coideario de Eloy Alfaro. Coral desde su ingreso en el periódico fue director y escritor. Camilo Destruge fue el primer redactor y colaborador de la plana mayor del radicalismo ecuatoriano. Otros periodistas de El Tiempo fueron Felicísimo López, José Peralta, José Domingo de Elizalde Vera y Juan Benigno Vela (Pérez, 1997).

En 1901 Luciano Coral Morillo se distanció del General Alfaro porque denunció el fraude electoral realizado para favorecer la candidatura oficial de Leonidas Plaza, a quien hizo cerrada oposición durante los cuatro años de su gobierno (1901-1905) desde las columnas de El Tiempo de Guayaquil (Pérez Pimentel, 2005a). En ese mismo año, en octubre, Coral fundó El Tiempo de Quito bajo el lema del diario "político, literario y noticioso" y se trasladó a vivir a la capital. En aquellos días El Tiempo estaba en una abierta oposición al gobierno de Lizardo García, a quien exigían la libertad de expresión (Corral Burbabo de Lara, 2006). Desde las columnas de este diario se habían 
publicado amplios comentarios acerca de la denuncia que hizo Eloy Alfaro en contra de Lizardo García, por el supuesto peculado en la renegociación de la deuda externa, cuando este último había sido comisionado fiscal en Londres, a pesar de que la justicia desligó las acusaciones, esta denuncia desacreditó el gobierno de Lizardo García afianzando de esta manera el golpe de Estado por parte de los alfaristas.

Su parcialidad con la causa liberal fue de mucha ayuda en la revolución que ya se estaba gestando. En 1906, cuando se dio la revuelta fueron perseguidos los redactores y encarcelado su director, pero esta suspensión duró muy poco. Se resolvió el 17 de enero de 1906, día del triunfo de la revolución. A fines de 1905 Luciano Coral adquirió un amplio edificio de tres pisos, donde trasladó los talleres y oficinas de su diario. El 22 de enero de 1912 dejó de publicarse El Tiempo de Quito. Sus herederos mantuvieron El Tiempo de Guayaquil bajo la dirección del periodista Manuel de Jesús Aguilar hasta 1923, año en que el diario cerró sus puertas (Delgadillo Avilés, 2007).

\section{Diraio La Patria}

En 1901 surgió un diario de la mañana con tendencia conservadora, La Patria. Su director era Juan Bautista Serrade oriundo de Latacunga. Su línea fue de total oposición al régimen. Su director, estaba vinculado con personajes conservadores y de altas jerarquías. Su hermano Filiberto, era presbítero y muy reconocido en la esfera clerical. En sus editoriales se hacía énfasis de la restauración de la moral pública pues, como opositores conservadores, el laicismo que era difundido por el alfarismo era un atentado contra la Iglesia católica a la que tanto defendían (Corral Burbabo de Lara, 2006).

En noviembre de 1905 La Patria expresó y lamentó la división de los liberales insistiendo en los desastres que esto iba a conllevar en la sociedad ecuatoriana. En los días de enero, en los que ya se acontecía la revuelta alfarista, La Patria mantuvo su crítica tenaz a estos hechos, sin dejar de lado su total y clara oposición contra Eloy Alfaro y su sistema de gobierno apegado a la dictadura. La Patria criticó al gobierno publicando en su editorial del 28 de abril de 1907: “¿Qué quiere, que hace aquí este viejo indigno de Eloy Alfaro, titulándose Presidente de la República cuando en realidad no es sino el jefe de una cuadrilla de bandidos?" (Corral Burbabo de Lara, 2006).

\section{El periódico vespertino La Ley y El Grito del Pueblo}

Junto a la oposición que realizaba La Patria al gobierno alfarista, en 1905 se sumó el periódico vespertino La Ley. Su directorio estaba conformado por personajes como: Clemente Ponce, Pablo Mariano Borja y Camilo Daste; hombres educados en la derecha más tradicional y conservadora. Estudios posteriores argumentan que el financiamiento de este diario estuvo a cargo de Manuel Jijón Larrea, promotor de la creación del Banco de Pichincha en 1906. En sus columnas se denuncia el fraude electoral que permitió la elección de Lizardo García. En la revisión de sus páginas hay la impresión de que lo financiaba era Manuel Jijón Larrea, promotor de la fundación del Banco Pichincha (Corral Burbabo De Lara, 2006).

El 22 de enero de 1892 Federico Reinel Rojas junto a José de Lapierre Cucalon y Luciano Corral fundaron el periódico El Grito del Pueblo (Pérez Pimentel, 2005) en cuyas columnas se encendió la chispa de la Revolución Liberal del 5 de junio de 1895. El Grito del Pueblo fue uno de los periódicos que se manifestó de una manera bastante crítica contra el gobierno del Dr. Luis Cordero por el escándalo internacional que produjo el tema del Buque Esmeraldas. Poco tiempo después este medio fue clausurado por orden del gobierno (De Janon Alcívar, 1948). Con la aparición del diario El Grito del Pueblo la prensa tomó un rumbo moderno porque fue el primer diario en Ecuador que se inició con el sistema de ilustración artística de los artículos referentes a los personajes que se destacaban en profesiones liberales. Este periódico fue el más amplio y detallado de Ecuador, contaba con corresponsales en las principales capitales de América y en las provincias más importantes de Ecuador. Sus publicaciones contenían, aparte de las columnas destinadas a los asuntos políticos, una sección de cablegramas, variedades, gacetilla y folletín. El Grito del Pueblo empezó a ser voceado en las calles por el primer grupo de canillitas o vendedores callejeros de periódicos que se conoció en Guayaquil. Federico Reinel Rojas fue el primer empresario periodístico que los contrató e instruyó. Este nuevo periódico funcionaba en el callejón Loja, la vivienda de propiedad de Camilo Echanique Morán y varios redactores de tinte liberal. Reinel tuvo que ausentarse por motivos de salud y permanecer largo tiempo en Lima. Durante su ausencia, el diario continuó su circulación hasta el número 6.102 que circuló el 30 de junio de 1911, en que apareció el último ejemplar. Entonces el periódico El Guante tomó su lugar. En este periódico se hizo durísima crítica al gobierno de la época. Al retornar a la ciudad Federico Reinel hizo acopio de elementos, y arreglando las cosas lo mejor posible, logró reeditar El Grito del Pueblo, agregando bajo su título, la palabra "Auténtico". Esta reaparición se produjo el día 13 de julio de 1916, con un formato mediano, cuatro planas a cinco columnas, editado en sus propios talleres (Pérez Pimentel, 2005).

\section{Los periódicos satíricos de El Buscapié a Fray Gerundio}

En 1905 en medio de tensiones políticas entre afines a Alfaro y sus detractores y conflictos dentro de la Iglesia por la pérdida de influencia en sus fieles, el panorama de la prensa era muy diverso. Los periódicos se dividieron claramente entre afines y opositores. Una prensa afín al pensamiento de Eloy Alfaro y su continuación en el poder, se oponía, a otra que estaba empeñada en apoyar el nuevo régimen imperante. A esta oposición de fuerzas que se produjo en el núcleo del liberalismo y en la prensa que le era afín, se agregaba otra muy notable: la que desde los días de la revolución en 1895, se autocalificó como liberal -defensora del nuevo régimen- y otra más conservadora que con lenguaje bastante burdo y tajante criticaba al régimen (Corral Burbabo de Lara, 2006). En estos días ya circulaba una prensa que se distanció de todos estos cansados procesos políticos, y empezó a comentar otros hechos, sobre todo relacionados con la vida cultural y a la producción intelectual. Este tipo de prensa fue afianzada por Manuel J. Calle, un cuencano que firmó muchos de sus artículos con los seudónimos de "Ernesto Mora" y "Enrique de Rastignac". Manuel J. Calle criticaba y satirizaba el pequeño mundo intelectual de aquel entonces en las columnas de su periódico con un talento innato: El Buscapié que salió a la luz en Quito el 4 de diciembre de 1905 con publicaciones sabatinas (Villaroel, 1994).

Aunque para Manuel J. Calle el objetivo de su periódico no era hacer daño a nadie como lo reiteraba en el lanzamiento del periódico, sus 
comentarios llenos de sarcasmo contradijeron sus palabras, pues con su estilo empezó a derribar a grandes de su época como Numa Pompilo Llona, Quintiliano Sánchez con su novela "Amar con desobediencia" de la cual escribió "como narración es un adefesio [...] como estudio de caracteres, costumbres y paisajes, una monstruosidad [...] y como obra de arte una solemne majadería, por decir lo menos", de esta manera Manuel J. Calle censuraba a la intelectualidad de esa época (Corral Burbabo de Lara, 2006).

Contradictorio a El Buscapié fue La Fronda Literaria con la misma línea literaria pero auspiciada por los liberales. También tenía su aparición los sábados, el primer número circuló el 18 de noviembre de 1905, y su objetivo también era el rescate de los valores de la literatura. Otro de los semanarios conocidos de esta época fue Fray Gerundio, tabloide que apareció el 9 de julio de 1898, que fue dirigido y financiado por Vicente Nieto. Bajo el lema "Cara patria, Carior Libertas". La publicación se mantuvo por varios años siempre en contra de los gobiernos liberales. Este semanario aparecía todavía en los últimos días de diciembre de 1905. Una anécdota de este semanario fue cuando la publicación se canceló por falta de papel y enterado de este hecho el presidente Plaza Gutiérrez, dispuso que se suministrara papel y tinta para su publicación. Quedaría en tela de duda si este hecho fue una habilidad política o una verdadera lección de democracia y respeto a los medios de comunicación.

Publicaciones como El Ecuador llustrado (1883), El Murciélago (1884), El Perico (1885), El Gavilán (1889), La argolla (1890) El Cordero (1891), La Marica (1894), El látigo (1895), entre otras, variaban artículos de denuncia en contra del régimen y crítica a instituciones como la Iglesia, con caricaturas encargadas de contar los complejos acontecimientos y protagonistas del escenario político de la época (Ibarra, 2006). En 1861, surge en Ecuador la primera revista ilustrada El Iris, editada por Juan Pablo Sanz, quien ha sido considerado como el pionero en establecer un taller de litografía en la capital. A finales del XIX se desplegó más ampliamente esta tecnología a nivel local, permitiendo el aparecimiento de diarios y revistas ilustradas de diversa índole que alternaban sus textos escritos con fotografías, grabados, diseños y caricaturas (Pérez Pimentel, 2005b). Se puede analizar las primeras experimentaciones con el dibujo humorístico hacia mediados del siglo XIX, en los trabajos de Juan Agustín Guerrero Toro; pero fue a partir de 1880, en el ambiente de las luchas entre liberales y conservadores que apareció con fuerza la caricatura en Guayaquil; especialmente de la mano de Juan Agustín Guerrero Toro, y luego, en los semanarios y quicenarios políticos que tendría una muy fugaz aparición. La presencia de la caricatura originó una nueva dinámica en el campo artístico que gestionó el uso del humor visual como herramienta crítica (Pérez Pimentel, 2005c).

En Quito, Enrique Terán Vaca, el 8 de diciembre de 1918, fundó junto a los escritores y artistas Jorge Carrera Andrade, Nicolás Delgado, Carlos Andrade (Kanela), Guillermo Latorre y Jorge Díez, Alberto Coloma Silva, Jorge Diez y Guillermo Latorre la revista humorística, semanal e ilustrada Caricatura. Enrique Terán Vaca colaboraba con numerosos dibujos que aparecieron en sus portadas y páginas interiores. En esta revista semanal también figuraron Nicolás Delgado, César y Jorge Carrera Andrade, Benjamín Carrión, Eduardo Batallas, Luis F. Rueda, Antonio Aguilera y otros. Caricatura marcó toda una etapa en el periodismo de Ecuador por sus dibujos y humor satírico. Para una ciudad que estaba demasiado anclada en las costumbres y tradiciones, era imposible aceptar las bromas de los redactores. Caricatura se creo para aceptarla con humor y para personas con criterio formado. Una escena con desnudo femenino dibujada por Nicolás Delgado atrajo críticas moralistas por parte del clero, pero el país siguió comprándola porque Caricatura era obra de jóvenes idealistas que apoyaban al arte en sus principales manifestaciones (Pérez Pimentel, 2005d).

El contenido de Caricatura incluía la publicación de partituras y caricaturas, artículos críticos diversos y una agenda musical. Pero lo que más llamaba la atención desde el punto de vista literario era que reseñaban y divulgaban las últimas tendencias provenientes de las revistas Cervantes, Grecia, Littérature, Nouvelle Revue Française, Ultra o Tableros, todas éstas editadas desde Europa. También surgieron los detractores incluso dentro de la misma publicación. El paso de "Caricatura" en Ecuador fue fructífero pero bastante corto pues solo se emitió hasta 1921 (Palacio, 2000).

\section{La consolidación del periodismo de empresa. De El Comercio a El Universo}

En medio de un ambiente de conflicto continuo de liberales y conservadores por el manejo del poder, la prensa tuvo una notoria presencia y a la vez representó las posiciones ideológicas. La prensa en esta época fue bastante efímera y sólo obedecía a fines propagandísticos del partido al que pertenecía. El poder siempre estuvo en manos de grupos conservadores y cuando explotó la revolución liberal los medios impresos que defendían su causa también salieron a la luz y como tal fueron numerosos pero dejaron de circular rápidamente. En la mayoría de periódicos el financiamiento corría por cuenta del propietario del medio y allegados. Este modelo permitió que la prensa fuera más accesible al pueblo. Con el ascenso al poder del estado liberal, en la cual el clero ya no tenía la misma influencia en los medios, la publicidad abrió una puerta muy importante de la prensa como empresa. Así aumentó de una forma considerable las revistas y periódicos promovidos principalmente por jóvenes empresarios.

En Ecuador, a inicios del siglo XX, la prensa tuvo menos obstáculos, porque la censura que ejercía el clero se abolió. Este hecho dio paso al surgimiento del periodismo como empresa, es decir con una organización, permanencia y con claros objetivos de obtener beneficio económico. A pesar del surgimiento casual y eventual de muchos periódicos y su desaparición fortuita los periódicos tuvieron desde un inicio intereses económicos y empezaron a constituirse como un negocio. Esto permitió su permanencia en el tiempo y su divulgación como medios masivos de comunicación. Los primeros representantes de la prensa y periodismo pertenecieron a un reducido grupo de intelectuales, que eran personas muy instruidas quienes expusieron sus ideas por medio de periódicos y revistas. Para pertenecer a este grupo burgués la mejor publicidad era la instrucción. El público burgués fue un público de lectores.

Una característica de la prensa a finales del siglo XIX e inicios del XX fue su combate que reflejaba la convicción de los ideales de los periodistas. Se defendió con valentía cada doctrina como fue el caso de El Telégrafo, El Tiempo o El Globo de tendencia liberal. Estos periódicos habrían apoyado la Revolución de 1895. En Ecuador los principales periódicos de aquella época fueron El Telégrafo, decano del diario nacional y El Comercio, fundado en 1906. La aparición de estos diarios originó que la fugacidad de los medios de comunicación se termine dando paso a la consolidación de un tipo de periodismo más estable (Destruge, 1984).

Después de 123 años de funcionar como una empresa privada, El Telégrafo pasó a ser propiedad del Estado Ecuatoriano, que lo declaró como el primer diario público del país tras ser adquirido por medio del fidecomiso de la Agencia de Garantía de Depósitos (AGD) No más 
Impunidad, que incautó el diario a Fernando Aspiazu, también dueño del banco del ya desaparecido Banco del Progreso (Checa Godoy, 2011). De esta manera se da paso a una nueva etapa en el desarrollo de la historia del periodismo ecuatoriano caracterizada por el surgimiento de mayor número de medios y sobre todo por la estabilidad prolongada en el tiempo.

El desarrollo de los medios de comunicación adquirió gran importancia porque a través de ellos se llegaba a un mayor número de personas y surgen entonces las primeras ediciones con espacios dedicados a la publicidad. Hecho que coincide con el desarrollo comercial y productivo del país. La prensa contribuía a generar un incipiente capitalismo. Los principales productos que se relacionaron con empresas e industrias aparecidas en Ecuador desde fines del siglo XIX como fueron las de harinas cigarrillos, cerveza, zapatos, tejidos y se anunciaban en los periódicos de la época. Otros productos ofertados fueron pianolas, vinos, medicinas, telas, llantas, libros, ropa, automóviles, jabones y perfumes (Vallejo, 1996).

A principios del siglo XX, aparecieron en Quito diario El Comercio, fundado el 1 de enero de 1906 por el Sr. Carlos Mantilla Jácome; y La Prensa, fundado en 1909 y dirigido por don Manuel María Sánchez; mientras en Guayaquil, el 28 de agosto de 1910 apareció El Guante, en el que escribieron y publicaron sus artículos personajes como César Borja Cordero, Pío Jaramillo Alvarado, José Falconí Villagómez, Wenceslao Pareja y Pareja, Miguel Neira, Eleodoro Avilés Minuche y Francisco Falquez Ampuero, entre otros. En 1913 aparece diario El Día, en 1920 diario La Tierra que se enfocó en la defensa de los obreros. Más tarde, el 16 de septiembre de 1921 -bajo la inspiración de don Ismael Pérez Pazmiño- hizo su aparición El Universo, en Guayaquil un periódico de mucho peso actualmente (Corral Burbabo de Lara, 2006).

\section{La huelga general de 1922}

La primera huelga general en Ecuador se dio en Guayaquil el 15 de noviembre de 1922. Dos días antes la ciudad ya se encontraba sin luz por la huelga que habían comenzado los trabajadores de la planta eléctrica. El día 14, en asamblea general los tipógrafos y canillitas de la ciudad decidieron que los diarios salgan por última vez al amanecer del día siguiente. La huelga fue convocada por la sindical Federación de Trabajadores Regional Ecuatoriana (FTRE) de inspiración anarcosindicalista.

La prensa registró los nombres de 80 hombres y 14 mujeres asesinados. A ellos se sumaron los recogidos sin identificar, que fueron lanzados a la ría o al zanjón del cementerio general. Hubo casi 200 heridos. Los diarios El Telégrafo y El Universo del 17 de noviembre reseñan que José Vicente Trujillo y Carlos Puig Vilazar, quienes estaban del lado de los obreros, instaron a la muchedumbre a una conducta mesurada. "Maldita sea la huelga, diríamos, si no nos constara que hay mucha justicia en los reclamos que la han motivado" (editorial de El Universo del 14 de noviembre de 1922).

La matanza del 15 de noviembre se hubiera evitado, si el gobierno resolvía a tiempo las peticiones de los trabajadores, y también si al mando de los gremios obreros no hubiera gente tan inescrupulosa que se aprovecha de la sensibilidad de personas con sed de justicia social.

\section{El diario El Día y el desarrollo de la prensa regional como El Mercurio de Cuenca}

Luis Robalino Dávila, junto a otros opositores al presidente Leónidas Plaza Gutiérrez como José Rafael Bustamante y Carlos Manuel Tobar Borgoño, fundan el periódico El Día el 1 de agosto de 1913. Bajo su dirección combatió duramente al gobierno. Posteriormente El Día fue adquirido por Ricardo Jaramillo, El Día fue el más izquierdista de los grandes rotativos ecuatorianos de los años treinta. El director Ricardo Jaramillo seguiría al mando del periódico desde el primer número hasta su clausura en 1946 (Pérez Pimentel, 2005d).

La mayoría de procesos políticos se han dado en Quito y Guayaquil donde hay una fuerte presencia de la prensa, que con el paso del tiempo se ha llegado a consolidar como empresa, lo que le permitió que sus periódicos llegaran a casi todos los rincones del país. No obstante la prensa regional, merece ser estudiada y analizada para posteriormente obtener conclusiones de la influencia que tuvo en los procesos políticos de Ecuador. El pionero del periodismo en el Azuay fue el Fray Vicente Solano y Vargas Machuca un profundo teólogo y sociólogo. Defendió tenazmente los derechos de Dios y la Iglesia (Pérez Pimentel, 2005d). En enero de 1828, publicó en Cuenca el primer periódico al que se denominó El Eco del Azuay que se mantuvo por 6 meses, salió en 26 números y en los primeros días de julio se suspendió su publicación. Su contenido fue diverso, desde la política a la filosofía y logró interesar a la élite del pensamiento de la unión de la Gran Colombia. Durante los años de la colonia Solano se destacó por su capacidad de reflexión y análisis, su estilo siempre fue crítico lo que le valió enemigos que si por un lado elogiaron su inteligencia, por otro impugnaron sus comentarios. Solano también despertó la desaprobación de la ciudadanía compatriota por su actitud contra la poetisa quiteña Dolores Veintimilla de Galindo, convertida en blanco de sus excesos verbales a través de panfletos y otros escritos.

El 22 de octubre de 1924, en Cuenca, se fundó el diario El Mercurio. El doctor Nicanor Merchán Bermeo dirigió el diario hasta 1956. Fue él quien modernizó y rediseñó el diario a su cargo. La primera década del periódico fue conducida por tres directores. El primero de ellos fue el doctor Carlos Aguilar Vásquez. Posteriormente le sucedió en el cargo el doctor Vicente Manuel Moreno Mora. Los dos se destacaron por su talento, visión del futuro y la habilidad para escribir. El tercer director fue el señor José Sarmiento Abad. Este periódico hace énfasis en afirmar que nunca ha pertenecido a ningún partido ni movimiento político (Pérez Pimentel, 2005f).

\section{A modo de conclusión}

Pasada la época de los grandes diarios que se forjaron a partir de ideales políticos o que se manifestaron neutrales a ellos, vino la época de creación de varios medios locales, regionales y nacionales cuyo principal objetivo es convertirse en verdaderas empresas periodísticas generadoras de rentabilidad para sus accionistas. Esto sin dejar de lado la información como derecho de los usuarios y como bien público.

La reciente Ley de Comunicación en Ecuador determinará el futuro de muchos medios de comunicación. El recorrido por la historia nacional nos obliga a recordar que los impuestos al papel y el recorte de publicidad pública pueden generar dificultades a los medios 
impresos. No hay que olvidar además la prohibición de dar declaraciones a los medios privados por parte de los personajes que ejercen cargos públicos de alto nivel. Planteado este escenario, y haciendo hincapé en las tecnologías de comunicación actuales, es muy difícil predecir el futuro de la prensa en Ecuador.

\section{Bibliografía}

AYALA MORA, E. (2008). "Los muertos del Florecimiento". Procesos, Revista Ecuatoriana de Historia. № 27, I Semestre.

BENÍTEZ, J.A. (2000). Los orígenes del periodismo en Nuestra América. Buenos Aires: Grupo Editorial Lumen.

CARRIÓN, B. (2008). El cuento de la patria (Breve historia del Ecuador). Quito: Libresa.

CHECA GODOY, A. (2011) "La batalla política y legal en Ecuador ante la creación de un sector estatal de medios informativos" Anuario Americanista Europeo, 9, 39-54.

CORRAL BURBABO DE LARA, F. (2006). Testigo del siglo: El Ecuador visto a través de diario El Comercio 1906-2006, Quito: Ediecuatorial.

DE JANON ALCÍVAR, E. (1948). El viejo luchador, su vida heróica y su magna obra, Tomo I, Quito: Editorial Abecedario llustrado.

DE VELASCO, J. (1981). Historia del reino de Quito, Caracas: Biblioteca Ayacucho.

DELGADILLO AVILÉS, L. (2007). Alfaro, la masonería y la historia, Guayaquil: Del autor.

DESTRUGE, C. (1984). Album biográfico ecuatoriano, Quito: Centro de Investigación y Cultura del Banco Central del Ecuador.

GÓMEZ ITURRALDE, J.A. (1998). Los periódicos guayaquileños en la historia, Guayaquil: Archivo Histórico del Guayas.

GÓMEZ ITURRALDE, J.A. (2006). Los Periódicos Guayquileños en la Historia (Vol. II), Guayaquil: Talleres gráficos del Archivo Histórico de Guayas.

IBARRA, H. (2006). Trazos del tiempo. La caricatura política en el Ecuador a mediados del siglo XX. Museo de la Ciudad. Serie de Documentos. Quito: Imprenta Flores.

IGLESIAS MATA, D. (1995). Eloy Alfaro. 100 facetas históricas. Manabí: Casa de la Cultura Ecuatoriana.

MAEDER, J.A. (2001). "Libros, Bibliotecas, control de lecturas e imprentas rioplatenses en los siglos XVI al XVIII”. Teología, XL, 77, 5-24.

MONTALVO, J. (1990). Las catilinarias, Quito: Editorial Libresa.

NUÑEZ ENDARA, P. (2001). Relaciones internacionales del Ecuador en la fundación de la República. Quito: Universidad Andina Simón Bolívar.

PALACIO, P. (2000). Obras completas, San José: Universidad de Costa Rica-ALLCA XX.

PÉREZ, J. (1997). Escritos y Testimonios, México: UNAM.

PÉREZ PIMENTEL, R. (2005). Diccionario Biográfico del Ecuador, Tomo VIII, Guayaquil: Foncultura.

PÉREZ PIMENTEL, R. (2005a). Diccionario Biográfico del Ecuador, Tomo I, Guayaquil: Foncultura.

PÉREZ PIMENTEL, R. (2005b). Diccionario Biográfico del Ecuador, Tomo IX, Guayaquil: Foncultura.

PÉREZ PIMENTEL, R. (2005c). Diccionario Biográfico del Ecuador, Tomo II, Guayaquil: Foncultura.

PÉREZ PIMENTEL, R. (2005d). Diccionario Biográfico del Ecuador, Tomo II, Guayaquil: Foncultura.

PÉREZ PIMENTEL, R. (2005e). Diccionario Biográfico del Ecuador, Tomo IV, Guayaquil: Foncultura.

PÉREZ PIMENTEL, R. (2005f). Diccionario Biográfico del Ecuador, Tomo XXII. Guayaquil: Foncultura.

PUNÍN, M.I. y RIVERA ROGEL, D.E. (2014). Las primeras huellas del periodismo ecuatoriano. De la censura a la libertad de prensa. RAEIC Revista de la Asociación Española de Investigación de la Comunicación. 2 (1): 116-123.

PONCE, P. (1990). Gabriel García Moreno, Quito: Editorial El Conejo.

REYES, O.E. (1970). Brevísima historia del Ecuador, Quito: Editorial ABC.

REYES CEDEÑO, J. (2000). "La Revolución del 5 de Mayo del 1895”. Cyberalfaro, 2. 
RIVERA ROGEL. D.E. (2012). "Breve historia de la prensa en Ecuador. El aporte de Loja". Chasqui, 119.

ROLANDO, C. (1947). Crónica del Periodismo en Ecuador (Vols. I 1792 - 1845), Guayaquil: Tip. de la Sociedad del Guayas.

ROLANDO, C.A. (1934). Cronología del periodismo ecuatoriano. Guayaquil: Tip. y Lit. de la Sociedad Filantrópica del Guayas.

TORRE REVELLO, J. (1991). El libro, la imprenta y el periodismo durante la dominación española, México: UNAM.

UGGEN, J.F. (2004). "Archer Harman y el ferrocarril del Sur". Procesos, Revista Ecuatoriana de Historia, 20.

VACA DEL POZO, T.N. (1941). El periodismo y las leyes de la imprenta: Periodismo funcional, Quito: Imprenta de la Universidad.

VALLEJO, G. (1996). Tres décadas de la publicidad en el Ecuador, Guayaquil: Grupo Offset Abad.

VILLAROEL, M. (1994). Historia Crítica de la Comunicación Social y el Periodismo en el Ecuador, Quito: Ediciones Conejo.

Cómo citar este artículo en bibliografías - How to cite this article in bibliographies / references:

MARÍN-GUTIÉRREZ, I.; RIVERA-ROGEL, D.; BARRAZUETA-MOLINA, P. (2016): "Desarrollo de la prensa en Ecuador. De la prensa ideológica a la empresa periodística". En Revista de la Asociación Española de Investigación de la Comunicación, vol. 3, número 5, pp. 88-95. 